\title{
Eficiência e deposição da aplicação de paraquat com adjuvantes e ângulo de inclinação das pontas sobre capim-colchão, falsa-serralha e caruru ${ }^{1}$
}

\author{
Efficiency and deposition of paraquat application with adjuvant and nozzles \\ inclination angle over Digitaria spp., Sonchus oleraceos and Amaranthus viridis
}

Cleber Daniel de Goes Maciel² ${ }^{2}$ Philipp Naoki Yokoyama Kondo ${ }^{3}$; André Prechlak Barbosa ${ }^{3}$; André Felipe Moreira Silva ${ }^{3}$; Matheus Akiyama Mendes ${ }^{3}$; Juliano Guilherme Sapia ${ }^{3}$; Andréia Paula Carneiro Martins ${ }^{4}$

Resumo - O uso de herbicidas de ação de contato com o paraquat normalmente necessita de maior atenção com aspectos de tecnologia de aplicação em relação aos de ação sistêmica. Com objetivo de avaliar a deposição e eficiência da aplicação de paraquat associado a adjuvantes e ângulos de inclinação das pontas de pulverização no controle de plantas daninhas, um experimento foi conduzido a campo em fatorial $2 \times 3+1$, com cinco repetições. Sete tratamentos foram constituídos por duas condições do herbicida Gramoxone ${ }^{\circledR}$ (paraquat, $150 \mathrm{~g}$ i.a. ha ${ }^{-1}$ ), utilizado com os adjuvantes Agral $^{\circledR}\left(0,1 \%\right.$ v/v) e Fulltec ${ }^{\circledR}(0,05 \%$ v/v; $)$, três angulações da inclinação da ponta de pulverização no sentido do deslocamento da aplicação $\left(+30^{\circ} ; 90^{\circ} ;-30^{\circ}\right)$ e uma testemunha sem aplicação. Os alvos naturais foram plantas de Digitaria spp., Sonchus oleraceos e Amaranthus viridis, e os alvos artificiais, tampas plásticas sobre a superfície do solo, sendo todos com 10 repetições. Não foram constadas diferenças significativas entre a deposição média da aplicação de paraquat com adjuvantes e ângulos da ponta de pulverização para $A$. viridis e lâminas de plástico. Para Digitaria spp. a deposição do paraquat com Agral $^{\circledR}$ foi superior ao Fulltec ${ }^{\circledR}$ utilizando-se ângulos de $+30^{\circ}$ e $90^{\circ}$. Para $S$. oleraceos houve aumento da deposição do paraquat com Fulltec ${ }^{\circledR}$ para o ângulo de $-30^{\circ}$. Todos os tratamentos controlaram eficientemente $(\geq 91,0 \%)$ as plantas daninhas aos 3 dias após aplicação, não havendo diferenças significativas em relação as condições estudadas.

Palavras-chaves: controle, tecnologia de aplicação, plantas daninhas

Abstract - The use of contact action herbicides with paraquat usually requires more attention with application technology aspects in relation to the systemic action ones. In order to evaluate the efficiency and deposition of paraquat application associated with adjuvant and nozzles inclination angle in weeds control, an experiment was conducted in field conditions using a factorial scheme $2 \times 3+1$, with five repetitions. Seven treatments were constituted by two

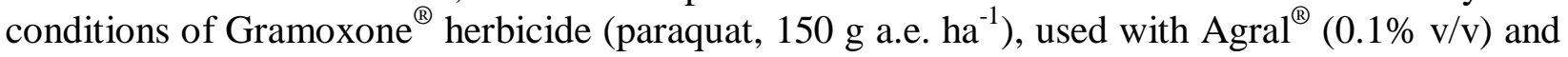

\footnotetext{
${ }^{1}$ Recebido para publicação em 02/02/2013 e aceito em 05/04/2013.

${ }^{2}$ Professor da Universidade Estadual do Centro Oeste (UNICENTRO), Campus CEDETEG, Curso de Agronomia, R. Simeão Varela de Sá, 03, CEP.: 85040-080, Guarapuava, PR. Email: cmaciel@unicentro.br (*autor para correspondência).

${ }^{3}$ Acadêmicos de Agronomia da Universidade Estadual de Maringá, Campus Regional de Umuarama, Estrada da Paca, s/nº, CP 65, 87501-970, Umuarama-PR.

${ }^{4}$ Técnica do Laboratório de Bioquímica da Universidade Estadual de Maringá, Campus Regional de Umuarama-PR.
} 
Fulltec $^{\circledR}(0.05 \% \mathrm{v} / \mathrm{v} ;)$ adjuvant, three nozzles inclination angle in direction of application offset $\left(+30^{\circ} ; 90^{\circ} ;-30^{\circ}\right)$ and a check without application. Natural targets were Digitaria spp., Sonchus oleraceos and Amaranthus viridis and artificial targets, plastic covers positioned over soil surface, with 10 repetitions. No significant differences were observed between application medium deposition of paraquat with adjuvant and nozzles inclination angles for $A$. viridis plastic covers. For Digitaria spp., paraquat deposition with Agral ${ }^{\circledR}$ was superior to Fulltec ${ }^{\circledR}$ using $+30^{\circ}$ and $90^{\circ}$ angles. For $S$. oleraceos there was increased in paraquat deposition with Fulltec ${ }^{\circledR}$ for $-30^{\circ}$ angle. All treatments presented efficient management $(\geq 91.0 \%)$ for weeds control at 3 days after application, with no significant differences regarding the studied conditions.

Keywords: control, application technology, weeds

\section{Introdução}

O controle de plantas daninhas é uma prática importante para obtenção de altos rendimentos em qualquer exploração agrícola. Entretanto, alguns aspectos técnicos relacionados à aplicação de herbicidas promovem e/ou mesmo garantem a eficiência de controle desejada, nas mais diferentes condições encontradas.

Entre estes aspectos, Theisen et al. (2004) mencionam os adjuvantes como sendo capaz de melhoram o ambiente da calda de pulverização e as condições para a proteção e absorção dos herbicidas. O uso de adjuvantes modifica as propriedades do líquido pulverizado, que podem influenciar tanto o processo de formação e espectro das gotas, como o comportamento destas em contato com o alvo (Miller \& Butler Ellis, 2000). Segundo Vargas \& Roman (2006), os adjuvantes podem alterar a característica de tensão superficial da solução, reduzindo o ângulo de contato das gotas com a superfície foliar das plantas daninhas. Maciel et al. (2011) constataram que os adjuvantes Agral $^{\circledR}, \quad$ Silwet $^{\circledR}$, Nimbus $^{\circledR}$, Naturl'Oleo $^{\circledR}$ e $\mathrm{Ag} \quad \mathrm{Bem}^{\circledR}$ elevaram significativamente o controle de Brachiaria decumbens, Brachiaria humidicola, Setaria geniculata e Sorghum bicolor, quando associado aos herbicidas de ação de contato, paraquat e MSMA.

Outros estudos enfatizam que o ângulo da barra pulverizadora inclinado na direção do deslocamento do pulverizador também pode proporcionar incrementos na deposição da aplicação sobre plantas daninhas, assim como na eficiência dos herbicidas (Friesen \& Wall, 1991; Shaw et al., 2000; Silva, 2000; Tomazela et al., 2006; Soares Filho, 2008). Raetano (2002), descreve em trabalho de revisão que resultados de pesquisas, conduzidas sob condições controladas e a campo, têm demonstrado que o posicionamento dos bicos a $30^{\circ}$ a favor do deslocamento em pulverizadores convencionais (sem ar) propiciam significativo aumento dos depósitos na superfície foliar de diferentes espécies vegetais.

O herbicida paraquat (1,1'-dimetil4,4'bipiridilio íon (dicloreto)), pertence ao grupo químico dos bipiridílios, e caracteriza-se por ter modo de ação de contato e atuar como inibidor do Fotossistema I; sendo recomendado para pulverizações em jato dirigido e área total para sistemas de plantio direto, na dessecação das plantas daninhas (Rodrigues \& Almeida, 2011). Segundo Silva et al. (2007), os herbicidas inibidores do Fotossistema I são rapidamente absorvidos pelas folhas principalmente na presença da luz, sendo que chuvas após 30 minutos de sua aplicação não mais influenciam a eficiência de controle das plantas daninhas. Portanto, a cobertura foliar é muito importante para herbicidas de baixa translocação, como é o caso do paraquat. Para isso, faz-se necessário a máxima eficiência na uniformidade de cobertura das aplicações para o controle eficiente sobre as plantas daninhas.

O objetivo deste trabalho foi quantificar a deposição e a eficiência da aplicação de paraquat associados a adjuvantes e ângulos de 
inclinação das pontas de pulverização no controle das plantas daninhas capim-colchão, falsa-serralha e caruru.

\section{Material e Métodos}

O experimento foi conduzido a campo, na Fazenda de Ensino e Pesquisa da Universidade Estadual de Maringá/ UEM, Campus Regional de Umuarama - PR, localizada a $23^{\circ} 47^{\prime} 28,4^{\prime \prime}$ de latitude Sul e $53^{\circ} 15^{\prime} 24,0^{\prime \prime}$ de longitude Oeste, em área com altitude média de aproximadamente $379 \mathrm{~m}$.

O solo da área experimental é classificado como LATOSSOLO VERMELHO Distrófico, textura arenosa (Embrapa, 2006), e o clima da região caracteriza-se como subúmido, com pouca deficiência hídrica e calor bem distribuído durante o ano, com estiagens no período de inverno, e médias anuais de temperatura e precipitação em torno de $24{ }^{\circ} \mathrm{C}$ e $1600 \mathrm{~mm}$, respectivamente (Iapar, 1994).

O delineamento experimental utilizado foi $\mathrm{o}$ de blocos casualizados com cinco repetições, onde sete tratamentos constituíram um fatorial $2 \times 3+1$, sendo duas as condições para o fator herbicida Gramoxone ${ }^{\circledR}$ (paraquat, $150 \mathrm{~g}$ i.a. ha $\left.{ }^{-1}\right)$, utilizado com os adjuvantes $\operatorname{Agral}^{\circledR}\left(0,1 \%\right.$ v/v) e Fulltec ${ }^{\circledR}(0,05 \%$ v/v; $)$, três as angulações da inclinação da ponta de pulverização no sentido do deslocamento da aplicação $\left(+30^{\circ} ; 90^{\circ} ;-30^{\circ}\right)$ (Figura 1$)$ e uma testemunha sem aplicação. As unidades experimentais foram representadas por área de $1,5 \times 5,0 \mathrm{~m}$, e principalmente composta por infestação de plantas daninhas constituídas por 57 plantas por $\mathrm{m}^{2}$ de capim-colchão (Digitaria spp.) em estádio de 1 a 2 perfilhos; 23 plantas por $\mathrm{m}^{2}$ de falsa-serralha (Sonchus oleraceos) em estádio de 3 a 4 folhas e 9 plantas por $\mathrm{m}^{2}$ de caruru (Amaranthus viridis) em estádio de 4 a 5 folhas.

Nas caldas de aplicação foi adicionado como traçador da pulverização o corante FD\&C-1, na concentração de 1500 ppm, conforme metodologia descrita por Souza et al. (2007) e Maciel et al. (2007). Palladini et al. (2005) mencionam que o corante FD\&C-1 não altera as características físicas da calda, podendo ser utilizado como traçador para simulação de aplicações de herbicidas.

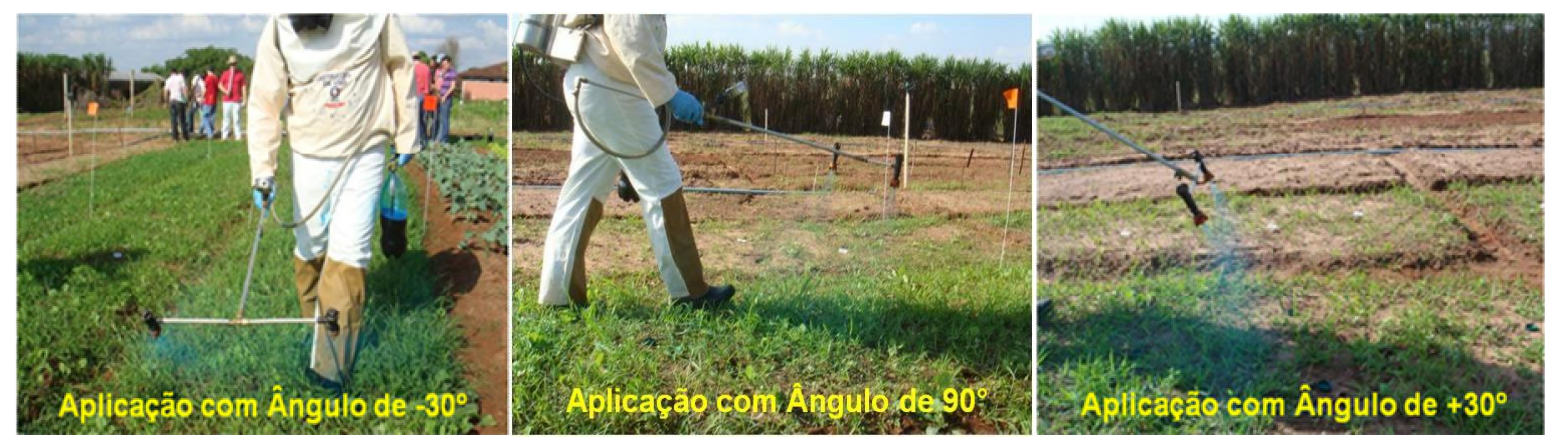

Figura 1. Representação esquemática da aplicação do herbicida+traçador com diferentes angulações de inclinação das pontas de pulverização no sentido do deslocamento da aplicação $\left(+30^{\circ} ; 90^{\circ} ;-30^{\circ}\right)$.

As aplicações foram realizadas em 15/05/2011, utilizando-se pulverizador costal pressurizado a $\mathrm{CO}_{2}$, equipado com duas pontas DG 110.02, espaçadas entre si em $0,5 \mathrm{~m} \mathrm{e}$ altura das plantas de $0,5 \mathrm{~m}$, e velocidade de 3,6 $\mathrm{km} \mathrm{h}^{-1}$, constituindo taxa de aplicação de $200 \mathrm{~L}$ $\mathrm{ha}^{-1}$ para todos os tratamentos. No início e final das aplicações realizada foi realizada entre 9:15 a 9:45 horas, onde a média da umidade relativa do ar, temperatura e velocidade dos ventos foram, respectivamente, de 67,3 a $68,0 \% ; 27,8$ a $27,3^{\circ} \mathrm{C}$ e 0,0 a $0,0 \mathrm{~km} \mathrm{~h}^{-1}$. 
A quantificação da deposição do traçante nos alvos naturais e artificiais foi por espectrofotometria (método colorimétrico), de forma semelhante a utilizada por Souza et al. (2007) e Maciel et al. (2007), com valores posteriormente transformados em $\mu \mathrm{L} \mathrm{g}^{-1}$ de matéria seca para os alvos plantas e $\mu \mathrm{L} \mathrm{cm}^{-2}$ para os alvos tampas plásticas. Como alvos naturais foram consideradas plantas das espécies capim-colchão (Digitaria spp.), falsaserralha ( $S$. oleraceos) e caruru (A. viridis), e como alvos artificiais, tampas plásticas sobre a superfície do solo, sendo em ambos os caso utilizados 10 repetições por tratamento.

As características físico-químicas, referentes ao $\mathrm{pH}$ e tensão superficial estática das soluções foram avaliadas, conforme metodologia descrita por Maciel et al. (2010), assim como para o controle das plantas daninhas aos 3 e 9 dias após aplicação (DAA), considerou-se uma escala percentual de notas, sendo $0 \%$ ausência de controle e $100 \%$ a morte das plantas (SBCPD, 1995).
Os dados foram submetidos à análise de variância pelo teste $\mathrm{F}$ e suas médias comparadas pelo teste $t$ a $5 \%$ de probabilidade.

\section{Resultados e Discussão}

Com relação às características físicoquímicas das caldas de aplicação, os adjuvantes Agral $^{\circledR}$ e Fulltec $^{\circledR}$ condicionaram reduções expressivas do $\mathrm{pH}$ e da tensão superficial estática para as soluções com paraquat (Figura 2). Essas características promovidas pelos adjuvantes podem maximizar a interação físico-química entre a formulação e a superfície das folhas, assim como reduzir a influência negativa apresentada em estruturas com ceras e tricomas das diferentes espécies de plantas daninhas, resultando em aumento da área de molhamento das gotas aplicadas. Entretanto, há poucas regras que governam a absorção de herbicidas e cada combinação entre herbicidas, espécies, formulações e ambiente constituem características próprias e únicas (Devine et al., 1993).

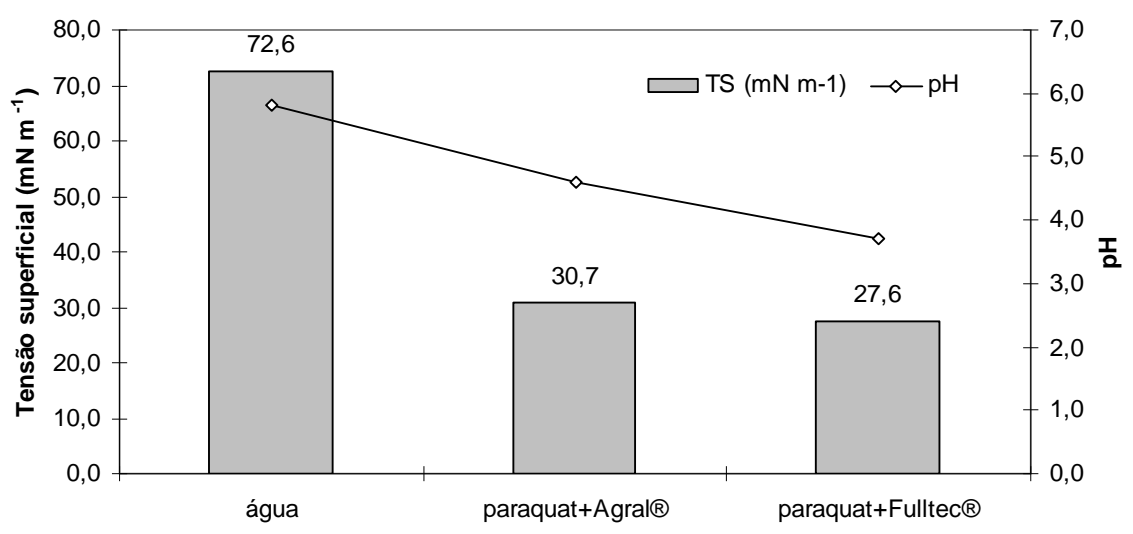

Figura 2. Tensão superficial estática e pHs das caldas de aplicação constituídas por paraquat $\left(\right.$ Gramoxone $^{\circledR}$ ) associado aos adjuvantes Agral ${ }^{\circledR}$ e Fulltec ${ }^{\circledR}$. Umuarama-PR, 2011.

Para deposição média da calda de pulverização do paraquat com os adjuvantes Agral $^{\circledR}$ e Fulltec ${ }^{\circledR}$ sobre as lâminas de plástico e para a espécie de planta daninha $A$. viridis não foram constadas diferenças significativas entre os adjuvantes e os ângulos de posicionamento da ponta na barra de aplicação (Tabela 1). A disposição horizontal dos referidos alvos talvez possa ter contribuído para a similaridade obtida na quantidade de depósito da aplicação. No entanto, cabe salientar que não foi encontrada nenhuma referência na literatura sobre estudos de deposição da calda de pulverização do herbicida paraquat em plantas daninhas, e/ou mesmo sobre alvos artificiais, que pudesse 
auxiliar na discussão dos resultados obtidos. Holloway (1970) menciona que a deposição e a uniformidade das aplicações de herbicidas estão diretamente relacionadas com as características tanto das folhas quanto da arquitetura das plantas.

Tabela 1. Deposição média da aplicação de paraquat $^{13}$ associado a adjuvantes utilizando diferentes ângulos das pontas de pulverização sobre as plantas daninhas Digitaria spp., Sonchus oleraceos e Amaranthus viridis, assim como em alvo artificial (tampas plásticas). UEM, Umuarama/PR, 2011.

\begin{tabular}{|c|c|c|c|c|c|c|c|c|}
\hline \multirow[t]{2}{*}{ Tratamentos } & Tamp & plásticas & $\begin{array}{r}\operatorname{Dig} \\
(\mu \mathrm{I}\end{array}$ & ria spp. & \multicolumn{2}{|c|}{$\begin{array}{l}\text { Sonchus oleraceos } \\
\left(\mu \mathrm{L} \mathrm{g}^{-1} \mathrm{MS}\right)\end{array}$} & \multicolumn{2}{|c|}{$\begin{array}{l}\text { Amaranthus viridis } \\
\left(\mu \mathrm{L} \mathrm{g}^{-1} \mathrm{MS}\right)\end{array}$} \\
\hline & Agral $^{(8)}$ & Fulltec ${ }^{17 / 2}$ & Agral $^{(\mathbb{1}}$ & Fulltec & Agral $^{(1)}$ & Fulltec & Agral $^{(8)}$ & Fulltec \\
\hline ângulo de $+30^{\circ}$ & 1,85 & 1,69 & $255,7 \mathrm{~A}$ & $206,1 \mathrm{~B}$ & $188,0 \mathrm{a}$ & $190,3 \mathrm{ab}$ & 211,6 & 203,8 \\
\hline ângulo de $90^{\circ}$ & 1,77 & 1,63 & $228,3 \mathrm{~A}$ & $184,6 \mathrm{~B}$ & $181,4 \mathrm{a}$ & $187,7 \mathrm{~b}$ & 187,3 & 194,0 \\
\hline ângulo de $-30^{\circ}$ & 1,74 & 1,61 & $240,5 \mathrm{~A}$ & $212,7 \mathrm{~A}$ & $203,0 \mathrm{a}$ & $224,5 \mathrm{a}$ & 216,1 & 229,3 \\
\hline testemunha sem aplicação & \multirow{2}{*}{\multicolumn{2}{|c|}{$0,439^{\mathrm{NS}}$}} & \multicolumn{2}{|r|}{-} & \multicolumn{2}{|c|}{ - } & \multicolumn{2}{|r|}{-} \\
\hline $\mathrm{F}_{\mathrm{c}}$ Ângulo da aplicação(Âng) & & & \multirow{2}{*}{\multicolumn{2}{|c|}{$\begin{array}{c}1,793^{\mathrm{NS}} \\
12,847^{* *}\end{array}$}} & \multicolumn{2}{|c|}{$3,027 *$} & \multicolumn{2}{|r|}{$2,963^{\mathrm{NS}}$} \\
\hline $\mathrm{F}_{\mathrm{c}}$ Adjuvante (Adj) & \multicolumn{2}{|c|}{$3,240^{\mathrm{NS}}$} & & & \multicolumn{2}{|c|}{$0,925^{\mathrm{NS}}$} & \multicolumn{2}{|r|}{$0,137^{\mathrm{NS}}$} \\
\hline $\mathrm{F}_{\mathrm{c}} \hat{A} \mathrm{Ang} \times \mathrm{Adj}$ & \multicolumn{2}{|c|}{$0,012^{\mathrm{NS}}$} & \multicolumn{2}{|c|}{$0,331^{\mathrm{NS}}$} & & $4^{\mathrm{NS}}$ & \multicolumn{2}{|r|}{$0,333^{\mathrm{NS}}$} \\
\hline $\mathrm{CV} \%$ & \multicolumn{2}{|c|}{18,28} & \multicolumn{2}{|c|}{19,70} & \multicolumn{2}{|c|}{20,62} & & 20,11 \\
\hline
\end{tabular}

Obs.: ${ }^{11} 0,1 \% \mathrm{v} / \mathrm{v} ;{ }^{12} 0,05 \% \mathrm{v} / \mathrm{v} ;{ }^{13} 150 \mathrm{~g}$ i.a. ha ${ }^{-1} ; \mathrm{MS}$ = matéria seca; - Médias seguidas de mesma letra maiúscula na linha e minúscula na coluna não diferem entre si pelo teste $t$ a $5 \%$ de probabilidade. $* *=\mathrm{P}<0,001 ; *=\mathrm{P}<0,05 ;{ }^{\mathrm{NS}}=$ não significativo.

Para Digitaria spp., a associação do paraquat com o adjuvante Agral $^{\circledR}(0,1 \%$ v/v) apresentou deposição significativamente superior em relação ao Fulltec ${ }^{\circledR}(0,05 \%$ v/v) apenas para aplicação com ângulos de $+30^{\circ} \mathrm{e}$ $90^{\circ}$ no sentido do deslocamento, onde os incrementos foram, em média, na ordem de $24,1 \%$ e $23,7 \%$, respectivamente. Maciel et al. (2011) também relataram que o adjuvante Agral $^{\circledR}$ elevou significativamente o controle das plantas daninhas Brachiaria decumbens, Brachiaria humidicola, Setaria geniculata e Sorghum bicolor, quando associado aos herbicidas paraquat e MSMA. Silva (2000) e Tomazella et al. (2006), mencionaram que ângulo de posicionamento da ponta de pulverização na barra de aplicação incrementou o depósito de calda aplicação sobre as espécies Cyperus rotundus e Brachiaria plantaginea, respectivamente, quando comparado com o ângulo de $90^{\circ}$. Soares Filho (2008), avaliando a deposição da pulverização em quatro espécies do gênero Commelina, também constatou que a angulação das pontas de pulverização em $+30^{\circ}$ pode incrementar a deposição e uniformidade da calda, mas são dependentes do volume de aplicação e espécie estudada.

Para a S. oleraceos não foi constatada interação significativa para condição adjuvante, tendo ocorrido apenas incremento significativo da deposição do paraquat com Fulltec ${ }^{\circledR}$ para aplicação com ângulo de $-30^{\circ}$, sendo o incremento em média da ordem de 19,6\%, em relação a aplicação em ângulo de $90^{\circ}$. Apesar das diferenças significativas encontradas na deposição da aplicação sobre as espécies Digitaria spp. e $S$. oleraceos, em relação ao adjuvante e/ou ângulo de aplicação, em termos de eficiência de controle aos 3 e 9 DAA (Tabela 2), não constatou-se diferenças significativas para as condições estudadas. Este fato certamente ocorreu em função da dosagem de paraquat (150 g i.a. ha ${ }^{-1}$ ) ter sido suficiente e adequada para controlar com elevada eficiência $(\geq$ $90,0 \%$ ) as plantas daninhas avaliadas, as quais apresentavam estágio de plântulas no momento da aplicação. 
Tabela 2. Controle (\%) das plantas daninhas Digitaria spp., Sonchus oleraceos e Amaranthus viridis, aos 3 e 9 DAA submetidas a aplicação de paraquat ${ }^{1 / 3}$ associado a adjuvantes utilizando diferentes ângulos das pontas de pulverização. UEM, Umuarama/PR, 2011.

\begin{tabular}{|c|c|c|c|c|c|c|c|c|c|c|}
\hline \multirow{3}{*}{ Tratamentos } & \multicolumn{4}{|c|}{ Digitaria spp. } & \multicolumn{4}{|c|}{ S. oleraceos } & \multirow{2}{*}{\multicolumn{2}{|c|}{ A. viridis }} \\
\hline & \multicolumn{2}{|c|}{$3 \mathrm{DAA}$} & \multicolumn{2}{|c|}{$9 \mathrm{DAA}$} & \multicolumn{2}{|c|}{$3 \mathrm{DAA}$} & \multicolumn{2}{|c|}{$9 \mathrm{DAA}$} & & \\
\hline & Agral $^{\circledR / 1}$ & Fulltec $^{\circledR / 2}$ & Agral $^{\circledR}$ & Fulltec $^{(B)}$ & Agral $^{\circledR}$ & Fulltec $^{(\circledR)}$ & Agral $^{\circledR}$ & Fulltec $^{(\circledR)}$ & Agral $^{\circledR}$ & Fulltec $^{\circledR}$ \\
\hline ângulo de $+30^{\circ}$ & $91,7 \mathrm{a}$ & $92,0 \mathrm{a}$ & $93,3 \mathrm{a}$ & $91,0 \mathrm{a}$ & $96,3 \mathrm{a}$ & $99,0 \mathrm{a}$ & 98,7 & 99,3 & 100,0 & 100,0 \\
\hline ângulo de $90^{\circ}$ & $91,0 \mathrm{a}$ & $91,0 \mathrm{a}$ & $92,7 \mathrm{a}$ & $91,7 \mathrm{a}$ & $98,7 \mathrm{a}$ & $98,7 \mathrm{a}$ & 99,7 & 100,0 & 100,0 & 100,0 \\
\hline ânguolo de $-30^{\circ}$ & $92,0 \mathrm{a}$ & $90,0 \mathrm{a}$ & $95,3 \mathrm{a}$ & $94,3 \mathrm{a}$ & $98,7 \mathrm{a}$ & $98,7 \mathrm{a}$ & 99,0 & 100,0 & 100,0 & 100,0 \\
\hline testemunha sem aplicação & \multicolumn{2}{|c|}{$0,0 \mathrm{~b}$} & \multicolumn{2}{|c|}{$0,0 \mathrm{~b}$} & \multicolumn{2}{|c|}{$0,0 \mathrm{~b}$} & \multicolumn{2}{|c|}{0,0} & \multicolumn{2}{|c|}{0,0} \\
\hline $\mathrm{F}_{\mathrm{c}}$ Ângulo da aplicação(Âng) & \multicolumn{2}{|c|}{$1980,875^{\text {** }}$} & \multicolumn{2}{|c|}{$1635,765^{* *}$} & \multicolumn{2}{|c|}{$3825,626^{\text {** }}$} & \multicolumn{2}{|r|}{-} & \multicolumn{2}{|r|}{ - } \\
\hline$F_{c}$ Adjuvante (Adj) & \multicolumn{2}{|c|}{$0,165^{\mathrm{NS}}$} & \multicolumn{2}{|c|}{$0,886^{\mathrm{NS}}$} & \multicolumn{2}{|c|}{$0,703^{\mathrm{NS}}$} & \multicolumn{2}{|r|}{ - } & \multicolumn{2}{|r|}{ - } \\
\hline $\mathrm{F}_{\mathrm{c}} \hat{A} \mathrm{ng} \times \mathrm{Adj}$ & \multicolumn{2}{|c|}{$0,271^{\mathrm{NS}}$} & \multicolumn{2}{|c|}{$0,173^{\mathrm{NS}}$} & \multicolumn{2}{|c|}{$0,703^{\mathrm{NS}}$} & \multirow{2}{*}{\multicolumn{2}{|c|}{$=$}} & \multicolumn{2}{|r|}{-} \\
\hline $\mathrm{CV} \%$ & \multicolumn{2}{|c|}{3,67} & \multicolumn{2}{|c|}{4,04} & \multicolumn{2}{|c|}{2.64} & & & \multicolumn{2}{|r|}{ - } \\
\hline
\end{tabular}

\section{Conclusões}

Não foram constadas diferenças significativas entre os adjuvantes e os ângulos da ponta de pulverização para deposição média do paraquat sobre lâminas de plástico e Amaranthus viridis. Para Digitaria spp., a deposição do paraquat com Agral $^{\circledR}(0,1 \%$ v/v) foi superior ao Fulltec ${ }^{\circledR}(0,05 \% \mathrm{v} / \mathrm{v}$; ) somente para os ângulos de $+30^{\circ}$ e $90^{\circ}$. Para Sonchus oleraceos foi constatado aumento da deposição do paraquat com Fulltec ${ }^{\circledR}$ para o ângulo de $30^{\circ}$. Em termos de eficiência de controle não constatou-se diferenças para as espécies e condições estudadas.

\section{Referências}

DEVINE, M.D.; DUKE, S.O.; FEDTKE, C. Physiology of herbicide action. London: Prentice Hall International, 1993. 441 p.

EMBRAPA. Centro Nacional de Pesquisa de Solos. Sistema brasileiro de classificação de solos. $2^{\text {a }}$ ed., Brasília: Embrapa Produção de Informação; Rio de Janeiro: Embrapa Solos, 2006. 306p.

FRIESEN, G.H.; WALL, D.A. Effect of application factors on efficacy of fluazifop-pbutyl in flax. Weed Technology, v.5, n.3, p.504-508, 1991.
HOLLOWAY, P.J. Surface factors affecting the wetting of leaves. Pesticide Science, v.1, n.1, p.56-63, 1970.

IAPAR - Instituto Agronômico do Paraná. Cartas climáticas do Estado do Paraná. Londrina: IAPAR, 1994. 49p. (IAPAR, Documento 18).

MACIEL, C.D.G. et al. Tensão superficial estática de misturas em tanque de glyphosate + chlorimuron-ethyl isoladas ou associadas a adjuvantes. Planta Daninha, v.28, n.3, p.673$685,2010$.

MACIEL, C.D.G; VELINI, E.; BERNARDO, R.S. Desempenho de pontas de pulverização em Brachiaria brizantha cv. MG-4 para controle de ninfas de cigarrinhas das pastagens. Engenharia Agrícola, v.27, n.1, p.66-74, 2007.

MILLER, P.C.H.; BUTLER ELLIS, M.C. Effects of formulation on spray nozzle performance for applications from groundbased boom sprayers. Crop Protecion, v.19, n.8, p.609-615, 2000.

PALLADINI, L.A.; RAETANO, C.G.; VELINI, E.D. Choice of tracers for the evaluation of spray deposits. Scientia Agricola, v.62, n.5, p.440-445, 2005.

RAETANO, C.G. Assistência de ar em pulverizadores de barra. Biológico, v.64, n.2, p.221-225, 2002. 
RODRIGUES, B.N.; ALMEIDA, F.S. Guia de herbicidas. 6. ed. Londrina: Ed. dos Autores, 2011.697 p.

SBCPD - Sociedade Brasileira da Ciência das Plantas Daninhas. Procedimentos para instalação, avaliação e análise de experimentos com herbicidas. $1^{\mathrm{a}}$ ed. Londrina: SBCPD, 1995. 42p.

SILVA, A.A.; FERREIRA, F.A.; FERREIRA, L.R. Herbicidas: classificação e mecanismos de ação. In: SILVA, A. A.; SILVA, J. F. Tópicos em manejo de plantas daninhas. UFV: Viçosa, 2007. p.58-117.

SILVA, M.A.S. Depósitos da calda de pulverização no solo e em plantas de tiririca (Cyperus rotundus L.) em diferentes condições de aplicação. 2000. 57 f. Tese (Doutorado em Agronomia/Agricultura) Universidade Estadual Paulista, Botucatu, 2000.

SOARES FILHO, S.I.B. Avaliação qualitativa e quantitativa na deposição de calda de pulverização em quatro espécies do gênero Commelina. 2008. 79 f. Dissertação (Mestrado em Agronomia/Agricultura) Universidade Estadual Paulista, Botucatu.

SOUZA, R.T.; VELINI, E.D.; PALLADINI, L.A. Aspectos metodológicos para análise de depósitos de pulverização pela determinação dos depósitos pontuais. Planta Daninha, v.25, n.1, p.195-202, 2007.

TOMAZELA, M.S. et al. Avaliação da deposição da calda de pulverização em função da densidade populacional de Brachiaria plantaginea, do volume e do ângulo de aplicação. Planta Daninha, v.24, n.1, p.183189, 2006.

VARGAS, L.; ROMAN, E.S. Conceitos e aplicações dos adjuvantes. Passo Fundo: Embrapa Trigo, 2006. 10 p. html. (Embrapa Trigo. Documentos Online, 56). Disponível em: <http://www.cnpt.embrapa.br/biblio/do/ p_do56.htm.> 